\title{
Minimally Invasive Non-Endoscopic Thyroidectomy: A Very High in the Neck Thyroidectomy Incision
}

\section{Dimitrios Linos}

\section{ABSTRACT}

We propose the adoption of a very high in the neck thyroidectomy incision, as high as the cricoid and/or thyroid cartilage, placed in an existing skin crease. This is a minimally invasive non-endoscopic thyroidectomy (MINET) approach that allows a safer, easier and more complete thyroidectomy.

Keywords: Incision, Neck, Thyroid.

How to cite this article: Linos D. Minimally Invasive NonEndoscopic Thyroidectomy: A Very High in the Neck Thyroidectomy Incision. World J Endoc Surg 2015;7(2):51-52.

\section{Source of support: Nil}

\section{Conflict of interest: None}

The thyroid gland anatomically lies high in the neck. Both thyroid lobes and the pyramidal lobe (PL) end up at the level or above the thyroid cartilage. Kocher's initial incision in the neck was vertical midline with lateral extension, in order to have better exposure of the upper pole of the thyroid lobe (Fig. 1). Later on he switched to his 100 years long known collar incision, placed $2 \mathrm{~cm}$ above the sternoclavicular joints, for a better cosmetic outcome! ${ }^{1}$

The Kocher incision was challenged in 1999 by the minimally invasive video-assisted thyroidectomy (MIVAT) technique, a much smaller in length incision $(2-3 \mathrm{~cm})$ but again placed at the same low in the neck level. $^{2}$ Since, the upper pole of the thyroid lobes could not be reached from this small incision located that low, the endoscopic camera and new instruments, including energy devices, were necessary.

In 2001, 'open' minimally invasive thyroidectomy (MIT) techniques appeared in the literature with small incisions ranging from 2.5 to $4.5 \mathrm{~cm}$, claiming equal cosmetic outcomes without the additional cost and risks of the MIVAT technique. ${ }^{3,4}$ The positioning of the incision was again placed at the Kocher incision level or slightly

\section{Professor}

Department of Surgery, Medical School, National and Kapodistrian University of Athens, Greece

Corresponding Author: Dimitrios Linos, Professor, Department of Surgery Medical School, National and Kapodistrian University of Athens Greece, Phone: 00302106255700, e-mail: dlinos@hms. harvard.edu higher, thus maintaining the inherent difficulties to easily reach the upper pole of the thyroid lobes and the tip of the PL.

In 2010, we first coined the term minimally invasive non-endoscopic thyroidectomy (MINET) to describe an 'open' MIT technique that uses a $2.5 \mathrm{~cm}$ incision placed much higher in the neck than the previously described approaches. ${ }^{5}$ The advantages of this incision which is placed at the level of cricoid or even thyroid cartilages along the usually existing skin creases, are:

- The full and more complete mobilization of the superior pole of the thyroid lobes, without leaving thyroid tissue behind.

- The recognition and extirpation of the whole PL that often ends up very high upto the hyoid bone.

- The easier recognition and preservation of the superior parathyroid gland.

- The protection of the external branch of the superior laryngeal nerve that descend on the surface of the inferior constictor to enter the cricothyroid muscle.

- The protection of the recurrent laryngeal nerve (RLN) as it enters the larynx going underneath the tubercle of Zuckerkandl.

Our 'audacity' to place a neck incision, as high as the cricoid and/or thyroid cartilage, along the almost always existing one or two natural skin creases, provided us with a surprisingly safer, easier and more complete thyroidectomy (Fig. 2).

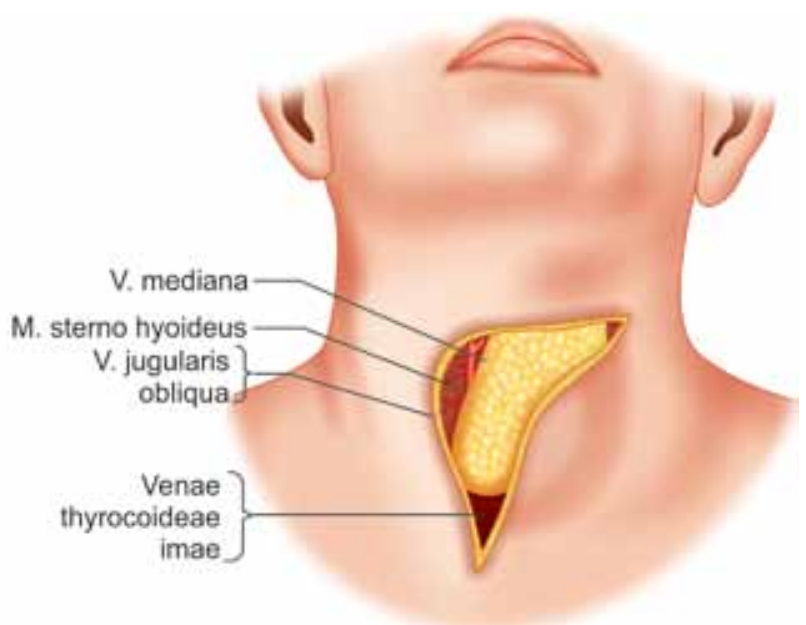

Fig. 1: Kocher's initial vertical incision with lateral extensions (from Kocher 1894) (with permission) ${ }^{1}$ 


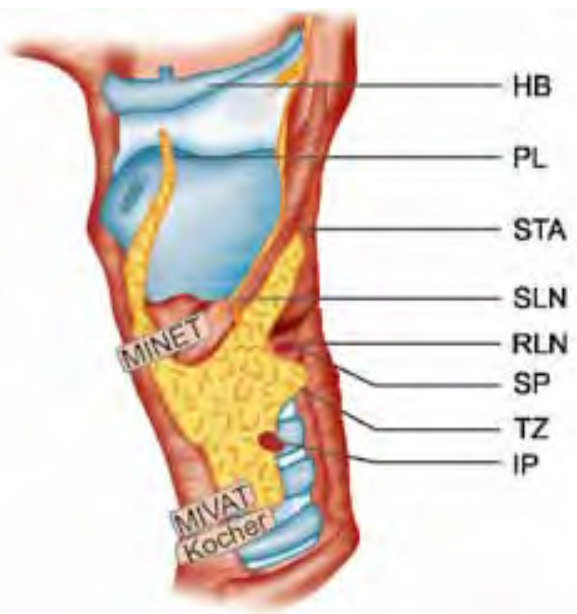

Fig. 2: Lateral view of the anatomy of the thyroid indicating the distance of three thyroidectomy incisions (Kocher, MIVAT and MINET) from the upper pole of thyroid and pyramidal lobes as well as the anatomic relations of the recurrent laryngeal nerve $(R L N)$, the external branches of the superior laryngeal nerve (SLN), the branches of the superior thyroid artery (STA), the pyramidal lobe $(\mathrm{PL})$ and its course to the hyoid bone $(\mathrm{HB})$, the superior $(\mathrm{SP})$ and inferior (IP) parathyroid glands and the tubercle of Zuckerkandl (TZ)

As far as the cosmetic outcome is concerned the less stretched high neck incision placed along an existing skin crease appears to offer smoother healing and a better cosmetic outcome. Using a validated patient scar assessment questionnaire (PSAQ) in a cohort of 691 patients that underwent MINET and conventional Kocher incision, we found similar patient satisfaction regardless of the procedure used. ${ }^{6}$ Similarly, when cosmesis and body image were examined in 250 patients, that had been operated with either MIVAT or open techniques no differences were found. ${ }^{7}$

Another advantage of our high in the neck approach is that we can extend the incision along the skin crease and accommodate large goiters. We can also perform synchronous lateral and middle compartment lymphadenectomies if necessary. Thus, the proposed high neck incision has no limitations regarding the size or histology of the thyroid.
Finally, the proposed MINET incision has no additional cost (endoscopic equipment or energy devices) and requires no additional training for the thyroid surgeon, since most of the required steps are similar to the ones of the traditional Kocher incision. ${ }^{8}$

\section{CONCLUSION}

We propose the adoption of a very high in the neck thyroidectomy incision placed in an existing skin crease, for a safer and more complete thyroidectomy. This high incision can be extended, if necessary, to accommodate all thyroid goiters and the synchronous performance of central or lateral lymphadenectomy.

\section{REFERENCES}

1. Hamberger B. History of thyroid surgery: the Kocher incision in D Linos and WY Chung, editors. Minimally Invasive Thyroidectomy: Springer, Berlin; 2012. p. 1-5.

2. Miccoli P, Berti P, Gonte M. Minimally invasive surgery for thyroid small nodules: preliminary report. J Endocrinol Invest 1999;22(11):849-851.

3. Park CS, Chung WY, Chang HS. Minimally invasive open thyroidectomy. Surg Today 2001;31(8):665-669.

4. Farzli GS, Sayad P, Ahdol, Cecchione RN. Minimally invasive non endoscopic thyroid surgery. J American College of Surgeons 2001;192(5):665-668.

5. Linos DA. Minimally invasive thyroid surgery. In: Frezza E, Gagner M, Li MKW, editors. International principles of laparoscopic surgery. Woodbury, CT: Cinemed; 2010. p. 531-537.

6. Linos D, Economopoulos KP, Kiriakopoulos A, Linos A, Petralias A. Scar perceptions after thyroid and parathyroid surgery: comparison of minimal and conventional approaches. Surgery 2013;153(3):400-407.

7. Bokor TH, Kniffner E, Kotrikova B, Billmann F. Cosmesis and body image after minimally invasive or open thyroid surgery. World J Surg 2012;36(6):1279-1285.

8. Linos D. Minimally invasive non endoscopic thyroidectomy: the MINET approach. In: Linos D, Chung WY, editors. Minimally Invasive Thyroidectomy. Springer, Berlin; 2012. p. 133-140. 